\title{
SOME METHODS USED IN ESTABLISHING THE MAXIMUM ALLOWABLE GONCENTRATIONS
}

\author{
E. I. LyUbLina
}

\section{Institute Gigieny Truda i Profzabolievanii, Leningrad, S.S.S.R.}

The continuous increase in the number of new chemical substances introduced into industry, or arising in the course of production, forces us to search for faster methods of establishing the maximum allowable concentrations (M.A.C.'s). It is obvious that the M.A.C. can only be one that does not have any harmful effects on the human organism in the course of full-time work of long duration.

It is, of course, impossible to design an experiment which could give an immediate answer to the problem. Only later can actual experience provide such a test, but, if the accepted M.A.G. has been set too high, the price paid for such an experiment is also high and might involve the loss of human life.

The results of regular medical examination of the workers, especially those working in an atmosphere filled with harmful substances, and other clinical and statistical data, can serve as material only for the correction of M.A.C.'s already determined. When a new substance is put into production, such information usually is not available. In these cases, only research can help.

Such experiments are of great importance when determining the M.A.C.'s for irritant gases and fumes.

We are forced to use experiments with animals in order to determine whether concentrations which have no harmful effect on humans exposed for short periods do have harmful effects in the case of long-term exposure.

The need for the rapid determination of the M.A.C.'s of substances newly introduced into industry does not allow these experiments to be carried out over the whole life-span. That is why, in such cases, it is not possible to reproduce the conditions which would apply to the human organism, even with respect to the overall length of exposure. Therefore it is all the more important to use highly sensitive methods, which make it possible to register even minor changes in the organism of the animal.

I will not discuss the various methods covered by the highly interesting and informative articles of Barnes and Denz, nor many of the methods used in the Soviet Union. I will discuss only various methods, worked out on the basis of a common principle.

In order to make this principle more comprehensible, let me remind you of the differences in the aims of the experiments carried out by a pharmacologist, and by a specialist in industrial toxicology. The pharmacologist is primarily interested in finding new drugs, and is, therefore, interested in the 


\section{E. I. LYUBLINA}

actual site of entry of the harmful substance into the human organism. The toxicologist, whose task it is to recommend the M.A.C. of vaporized substances in the air, is more interested in quantitative relations. It is essential for him to know at what concentration and exposure the substance is harmful for the organism, regardless of the mechanism of these effects. Knowledge of the mechanism is, nevertheless, important for toxicology, of course, and is necessary also for resolving other problems, such as the early diagnosis of poisoning and working out of therapeutic methods of fighting such poisoning. These latter problems also require further study.

In view of this difference of aims followed by the researchers, the pharmacologist uses special methods which allow him to note the changes in bodily organs and nerve centres which are caused by the substance under examination; the pharmacologist's main method is pharmacological analysis. An expert in industrial toxicology, on the other hand, tends to follow the path of synthetic research. He tries to answer the question whether or not any changes have occurred as a result of exposure to the poison, rather than determining where such changes occurred. This consideration forms the basic factor when searching for integral pointers about the state of the organism. The principle of how to use these pointers is known-it is the basic fact that the individual functions of the body are mutually interrelated.

Whether or not the changes in the organism caused by the effect of toxic substances are localized is sometimes unknown to the toxicologist, but if such changes exist at all they will undoubtedly affect the whole organism.

These ideas were described in detail by the founder of Soviet industrial toxicology, Professor Lazarev, at the last congress of experts on hygiene of the Soviet Union, held at Leningrad in 1956.

Data about the functional state of the central nervous system as well as the mutual relations of neural centres and all other organs may be used as integral pointers in view of the fact that the whole nervous system reacts to any harmful influence.

Clinical observations have taught us that the first signs of the influence of various substances are shown by the nervous system. Usually non-specific neurotic symptoms appear before the specific influence of the poison is established.' It is the research into a wide range of changes in the functional state of the central nervous system which is a typical trait of the methods used in the Soviet Union in experimental research for the determination of the M.A.C.'s of toxic substances in industry.

Recently, many industrial toxicologists in the Soviet Union have used this approach in the examination of higher nervous functions. These methods have, on the one hand, been used to establish the threshold concentration which affects the conditioned reflex with a single exposure. Such experiments are highly important for comparative evaluation of low-level toxicity, which is of basic importance for the determination of M.A.C.'s. On the other hand, the functions of the higher nervous activity have been examined also during chronic experiments. In a number of cases the exceptional sensitivity of this method has been proved. Izmerov, for instance, found out that rats, exposed to the daily effects of petrol fumes (Galoshes mark) having a concentration of $0.1 \mathrm{mg} / \mathrm{l}$., towards the end of the third month showed desinhibition and later a disturbance of quantitative relations of intensities 
of stimulus and response (to use the terminology of Vvedensky, they showed a balancing and paradoxical phase of parabiosis).

Our industrial toxicologists during their experiments in determining the effects of a toxic substance on the higher nervous activity have rarely used the classical method of inducing salivation in dogs as a conditioned reflex. Kotlyarevskii's method, which makes a rat press against a glass door to get food, is used more often, as well as the method of run reactions with alimentary or nociceptive reinforcement. Pravdin used the method of a run reaction with food incentive for the first time in industrial toxicology, and the pain incentive was used by Alexandrov and Tsibina.

To make the investigation of the higher nervous functions even more sensitive, a method of "working-out" of conditioned reflexes under daily exposure is being used. Golubiev proved in his experiments that the working-out of positive conditioned reflex with mice exposed to trichloropropane poisoning delayed the appearance of rather than caused a disturbance of an established conditioned reflex. Working-out a reflex under chronic effects of the poison has the advantage of not demanding a longterm preparation of the animal.

Although the methods of examination of these higher nervous functions are very sensitive, they cannot be considered to be universal and so enable us to discover the harmful after-effects of being exposed to poison as soon as possible. For example, the threshold concentration of formaldehyde which disturbs the higher nervous function of rabbits has been proved, according to Van Wein Yan, to be twice as high as the concentration which causes, by its irritant effect, a sudden slow-down of the breathing, which can appear at a concentration as low as $0.0075 \mathrm{mg} / \mathrm{l}$. The work done by Spynu (Kiev Institute of Industrial Hygiene and Occupational Diseases) has proved that a cat injected with $3 \mathrm{mg} / \mathrm{kg}$ of " thiophos" showed only the initial changes of conditioned reflex activity accompanied by a considerable drop in the cholinesterase activity ( $30-40$ per cent). The recovery of cholinesterase occurred much later than that of the higher nervous activity.

Today it is difficult to choose between the methods of examining conditioned reflexes for the establishment of M.A.C.'s. Undoubtedly the comparison of toxicity at this level has to be carried out during actual exposure and not after its termination. In the latter case a transient change, or a fast excretion of substances from the organism, could cause serious mistakes.

In chronic poisoning experiments, it becomes necessary to test the higher nervous functions before applying the poison, in order to be able to determine the effect of earlier doses and thus to avoid attributing the entire effect to the most recent exposure. The intricate method of conditioned reflexes does not usually prove successful in chronic poisoning experiments. For instance, Shachbazian did not notice a disturbance of the differentiation in cats suffering from strong hexafloran poisoning. In cases of heavy poisoning (when marked ataxia was exhibited), the higher nervous activity of the animals under observation was not disturbed. Apparently in these chronic experiments, the cerebral cortex, whose basic function is to adjust the organism to its environment, has itself adjusted to these poisons, even when the other functions have obviously been damaged and there is absolutely no doubt about the actual poisoning. 


\section{E. I. LYUBLINA}

An analysis of unconditioned motor reflexes, e.g., the flexor reflex in rabbits, is currently used by a number of our laboratories to determine the threshold concentration of the poison in short-duration experiments. In applying this method, an electrode is inserted into the folds of the skin on the lower third of the rabbit's tibia. The time from the onset of stimulation to the development of a certain reflex tension of the muscle, e.g., $1 \mathrm{~kg}$, is registered. This method has been used in our laboratories quite often when studying chronic poisoning. Depending on the nature of the poison under examination, either a gradual decrease of the reflex activity of the rabbit was observed (increase in the time of development of the reflex tension and decrease in its rate) or a decrease in the time and an increase in the rate of the reflex tension, which prove the increased excitability of the central nervous system, were obtained. The toxicologists testing the unconditioned motor reflexes may use not only rabbits, but rats, guinea-pigs and mice as well.

The changes in the ability of the central nervous system to summate subliminal stimuli were applied many times to determine the effects of small amounts of toxic substances upon various animals.

Our laboratories have in recent years applied these long- and short-term tests for determining the ability of summation of subliminal stimuli successfully on mice and rats.

Another method used in determining the effects of poison on the nervous system is measurement of a subordination chronaxie of the motor nerve.

Among the integral methods often used, testing not only the functional state of the nervous system, is the swimming test. The maximum time of the forced swimming is characteristic for the performance of an animal whose organism has suffered from a harmful interference, regardless of the function which has been attacked by this interference.

The conditions for applying this method in industrial toxicology (the temperature of the water, the load, etc.), were elaborated by Rylova in our laboratories.

The swimming of mice is usually used towards the end of a chronic experiment, by testing mice of the experimental and control samples at the same time.

Experience from functional diagnosis and physiology of work in man led to the idea that it would be well to use various types of functional experiments for an early diagnosis of the harmful effects of small amounts of low concentrations of poison on the organism. For this purpose we are using orthostatic tests on rabbits, tests in which breathing is made difficult, and tests to measure the time necessary for the recovery of the breathing frequency after a certain amount of exhaustion in guinea-pigs and mice.

In a typical orthostatic experiment, the rabbit was attached to a special apparatus which could be turned easily into a vertical position and fastened there. The following indicators were observed: increased heart rate, decrease of the systolic pressure and decrease of the breathing frequency, which are usually found in rabbits in a vertical position, as well as the time necessary to restore the normal functions when the rabbit was returned to a horizontal position. The duration of the vertical position was limited to 3 minutes. 
To test breathing difficulties a rabbit kept in its normal position was given a breathing mask. The valve for the exhaled air was attached to a gas counter and to the valve for incoming air. After normal breathing was restored, a rubber tube with a glass tube inside was inserted for one minute. The resistance when the air was inhaled through the glass tube was equivalent to $40 \mathrm{~mm}$ on a water manometer, at a uniform air circulation rate of 11./min. Changesin the lung ventilation, changes of the frequency and depth of breathing, and the nature and time necessary for the restoration of the breathing after the device has been removed, were examined.

Functional tests with dyspnoic rabbits and guinea-pigs (the latter run in a circle for one minute), were used over a period of four months, six hours a day, using carbon dioxide at a concentration of $0.03 \mathrm{mg} / \mathrm{l}$. (Gadaskina, Lyublina, Rylova). During the first month of these experiments in rabbits and guinea-pigs, certain variations of the above-mentioned function tests were observed.

In some other experiments a test of the recovery of the normal forward motion in mice after placing them for a short time in a centrifuge was successfully used. Experimental and control animals were rotated at the same time.

In order to determine the so-called "hidden effects" of poison on the organism, it is preferable to place the animals in an inferior environment, especially adjusted for this purpose. Thus it is usually possible to determine the chronic effects of small concentrations of poison, which otherwise could not be determined without increasing the claims on the organism of the animal. By an inferior environment we mean a qualitative and quantitative restriction of food, infection of the animals, frequent physical exertion, etc.

Using pregnant female or rapidly growing young animals makes it possible to establish the effects of toxic substances in doses and concentrations which otherwise could not be determined.

In the Soviet Union, methods of testing the functional state of the central nervous system are used to determine the threshold concentrations of various poisons (methods of conditioned and unconditioned reflexes). In chronic poisoning experiments, great importance is attached to the "integral indicators" of the overall condition of the animal, especially in various functional experiments, during research on animals which are placed in artificially produced inferior conditions. The high degree of sensitivity of the above quoted methods entitles us to consider developing similar methods which would be of value in meeting the needs of industrial toxicology.

We hope that our brief and far from complete contribution on methods used in Soviet laboratories of Industrial Toxicology may arouse the interest interest of our colleagues from other countries. 